\title{
Mathematical modelling of ground temperature with suction velocity and radiation
}

\author{
C. Nwaigwe \\ Department of Mathematics/Computer Science, Rivers State University of Science and \\ Technology, Port Harcourt, Nigeria
}

\begin{abstract}
This paper investigates the problem of ground temperature under a vegetative cover with time dependent suction and radiative heat transfer. By assuming that the ground radiates heat from its' surface and that the radiative heat flux takes a differential form which follows that of Tarkhar et al [1], the problem was formulated. By imposing a regular time dependent perturbation, the problem was solved. The numerical results and graphs were computed using the software, Mathematica. Results revealed that individual and simultaneous increase in flow parameters caused decrease in the temperature.
\end{abstract}

Keywords: Ground temperature, radiation, velocity

\section{INTRODUCTION}

The land surface plays an important role in partitioning net solar and downward longwave radiation into emitted longwave radiation, latent and ground heat fluxes. It also plays an important role in partitioning precipitation into runoff and evaporation. Accurate estimation of ground heat flux is usually translated into biases in latent and sensible heat fluxes and surface temperature [2].

Prediction of the diurnal cycle of ground heat flux is especially important for applications to numerical weather prediction models due to effects on surface air temperature. Field measurements have shown that ground heat flux at the surface is affected by heat stored or released in the soil layer between the surface and the measurement level. Therefore, another important aspect of estimating the ground heat flux is by estimating the heat storage [3].

The exclusion of radiation attenuation through the canopy layer causes an overestimation of ground heat flux during the daytime and underestimated at night, so combined effects of the heat storage and net radiation through the canopy may result in cancelling of errors to some extent.

Generally, flows in porous media have had much attention from researchers due to their numerous applications for instance Isreal-Cookey et al [4], [5], [6], Soundalgekar [7], [8], [9] and Alagoa [10] among many others have been investigating the problems of fluid flows in porous medium with suction velocity in which radiative heat plays an important role. Also
Choudhury et al [11] have studied the analysis of soil heat flux under a growing wheat crop.

The present work is aimed at investigating the problem of heat energy flow in ground with vegetative cover and radiative heat transfer. Hence, the problem is formulated in section 2, solved in section 3 and results discussed in sections 4 and 5 .

Formulation of the problem: Consider the heat flow in a porous medium (ground). The direction of flow is in the $z^{\prime}$-axis (see figure 1). The surface of ground is maintained at a constant temperature, $T_{w}^{\prime}$. Assume that heat flows into the ground through the surface and that the ground temperature is high so that radiative heat transfer is significant.

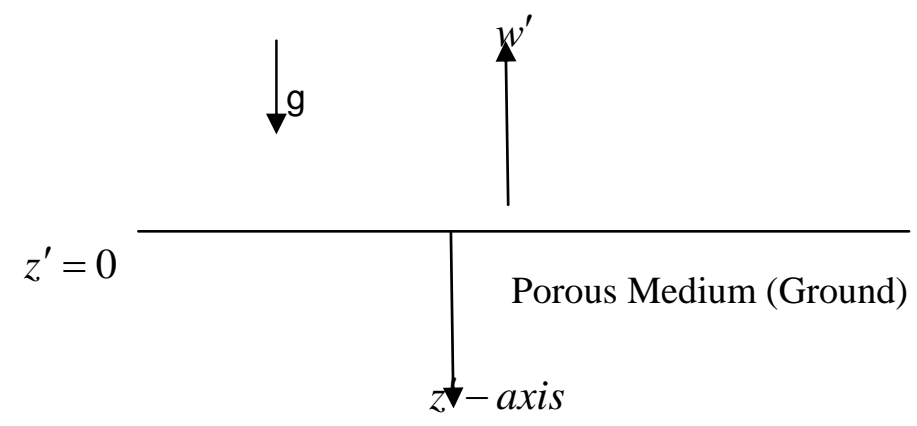

(Direction of heat flow)

Fig 1: The physical model and coordinate system of the problem. 
We also impose the following conditions that (i) the compositions of ground is homogeneous so that the specific heat capacity $c_{p}$, the thermal conductivity $k$ and the density $\rho$ are all constants and do not depend on the position $z^{\prime}$ or the temperature $T^{\prime}$ (ii) ground is not elastic so that the distance between $z^{\prime}$ and $z^{\prime}+\Delta z^{\prime}$ does not change and (iii) heat does not flow across the horizontal direction so that the temperature of any horizontal point at any time is equal.

Under these assumptions, the flow is governed by the following equations;

Continuity Equation:

$\frac{\partial \rho}{\partial t^{\prime}}+\frac{\partial\left(\rho w^{\prime}\right)}{\partial z^{\prime}}=0 \Rightarrow \frac{\partial w^{\prime}}{\partial z^{\prime}}=0$

Energy equation:

$\frac{\partial T^{\prime}}{\partial t^{\prime}}+\frac{w^{\prime} \partial T^{\prime}}{\partial z^{\prime}}=\frac{\kappa}{\rho C_{P}} \frac{\partial^{2} T^{\prime}}{\partial z^{\prime 2}}-\frac{1}{\rho C_{P}} \frac{\partial q_{r}}{\partial z^{\prime}}$

Where $\frac{\partial q^{\prime}}{\partial z^{\prime}}$ is the radiative heat flux, $w^{\prime}$ is velocity of fluid flow.

And the boundary conditions

$T^{\prime}=T_{w}^{\prime}$ on $z^{\prime}=0$ (wall temperature) and

$T^{\prime} \rightarrow 0$ as $z^{\prime} \rightarrow \infty$

From the continuity equation, $w^{\prime}$ is not a function of $z^{\prime}$ so it is a function of time only and following IsrealCookey et al [4] we set

$w^{\prime}=-w_{0}^{\prime}\left(1+\varepsilon A e^{i \omega^{\prime} t}\right)$

Where $w_{0}^{\prime}$ is initial suction velocity, $\omega^{\prime}$ is the frequency of oscillation and the negative sign indicates that suction is towards ground surface. A and $\varepsilon$ are very small positive parameters such that their product is very far less than one i.e. $\varepsilon A<1$. Again, we assume that the ground is an optically thin environment and so by Tarkhar et al (1996) radiative flux takes the form

$\frac{\partial q^{\prime}}{\partial z^{\prime}}=4 \alpha^{2}\left(T^{\prime}-T_{\infty}\right)$

Where $\alpha$ absorption coefficient

Hence equation (2) becomes

$\frac{\partial T^{\prime}}{\partial t^{\prime}}+\frac{w^{\prime} \partial T^{\prime}}{\partial z^{\prime}}=\frac{\kappa}{\rho C_{P}} \frac{\partial^{2} T^{\prime}}{\partial z^{\prime 2}}-\frac{4 \alpha^{2}}{c_{p}}\left(T^{\prime}-T \infty\right)$

Introducing the following non-dimensional parameters:

$$
\begin{aligned}
t & =\frac{w_{0}^{\prime 2}}{4 v}, \\
z & =\frac{w_{0}^{\prime} z^{\prime}}{v}, \\
\omega & =\frac{4 v}{w_{0}^{\prime}} \omega^{\prime}, \\
\theta & =\frac{T^{\prime}-T \infty}{T w^{\prime}-T \infty}, \\
v & =\frac{\mu}{\rho} \\
R^{2} & =\frac{4 \alpha^{2}}{\rho c_{p} \kappa w_{0}^{\prime 2}}\left(T_{w}^{\prime}-T \infty\right), \\
P_{r} & =\frac{\mu c}{\kappa}
\end{aligned}
$$

Equation (4) after simplification, becomes

$\frac{1}{4} P_{r} \frac{\partial \theta}{\partial t}-P_{r}\left(1+\varepsilon A e^{i w t}\right) \frac{\partial \theta}{\partial z}=\frac{\partial^{2} \theta}{\partial z^{2}}-R^{2} \theta$

Therefore, the problem becomes $\frac{1}{4} P_{r} \frac{\partial \theta}{\partial t}-P_{r}\left(1+\varepsilon A e^{i w t}\right) \frac{\partial \theta}{\partial z}=\frac{\partial^{2} \theta}{\partial z^{2}}-R^{2} \theta$

Subject to

$\theta=1$ on $z=0$ and

$\theta \rightarrow 0$ as $Z \rightarrow \infty$

Where $R=$ radiative parameter, $P_{r}=$ Prandtl number, $A=$ suction parameter.

\section{METHOD OF SOLUTION}

Equation (5) is a partial differential equation and since $\varepsilon$ is small, we adopt a time dependent perturbation method for the flow variable and assume a solution of the form.

$\theta(z, t)=\theta_{0}(z)+\theta_{1}(z) \varepsilon e^{i w t}$

Putting equation (7) into (5) and (6) and simplifying results, we find the order one equations

$\frac{d^{2} \theta_{0}}{d z^{2}}+P_{r} \frac{d \theta_{0}}{d z}-\theta_{0} R^{2}=0$

Subject to $\theta_{0}=1$ on $z=0$ and

$$
\theta \rightarrow 0 \text { as } z \rightarrow \infty
$$

And also obtain the first order $0(\varepsilon)$ equations:

$$
\frac{d^{2} \theta_{1}}{d z^{2}}+P_{r} \frac{d \theta_{1}}{d z}-\left(\frac{1}{4} P_{r} i \omega+R^{2}\right) \theta_{1}=P_{r} A \frac{d \theta_{0}}{d z}
$$


Am. J. Sci. Ind. Res., 2010, 1(2): 238-241

Subject to $\theta_{1}=0$ on $\mathrm{Z}=0$

$$
\theta_{1} \rightarrow 0 \text { as } \mathrm{Z} \rightarrow \infty
$$

Solving the system (8a-8b) and putting the result in $(9 a-9 b)$ we have,

$$
\theta_{0}(z)=e^{-m_{3} z}
$$

And $\theta_{1}(z)=N\left(e^{-m_{3} z}-e^{-m_{6} z}\right)$

Hence

$$
\theta(z, t)=e^{-m_{3} z}+N\left(e^{-m_{3} z}-e^{-m_{6} z}\right)
$$

Where $m_{3}=\frac{1}{2}\left[P_{r}+\sqrt{P_{r}^{2}+4 R^{2}}\right]$

$$
\begin{aligned}
& m_{6}=\frac{1}{2}\left[P_{r}+\sqrt{P_{r}^{2}+i \omega P_{r}+4 R^{2}}\right] \\
& \mathrm{N}=\frac{P_{r} A m_{3}}{m_{3}{ }^{2}-P_{r} m_{3}-Q} \\
& Q=\frac{1}{4} i \omega P_{r}+R^{2}
\end{aligned}
$$

\section{RESULTS AND DISCUSSION OF RESULTS}

In the previous sections, the problem of ground temperature in the presence of radiation and timedependent suction has been formulated and solved. The numerically computed and graphical results are also presented with the boundary condition $Z \rightarrow \infty$ being approximated by $Z_{\max }=2$, which is sufficiently large for the temperature to approach the free steam value.

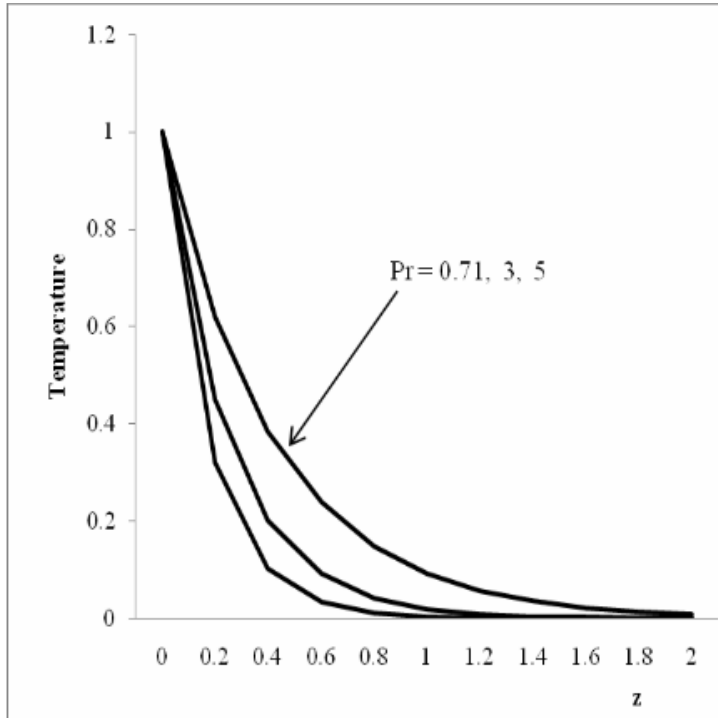

Fig. 2: Temperature profiles for $\varepsilon=0.01, \omega=2, t=1, A$ $=0.1, R=2$ and different values of

Prandtl number, Pr.

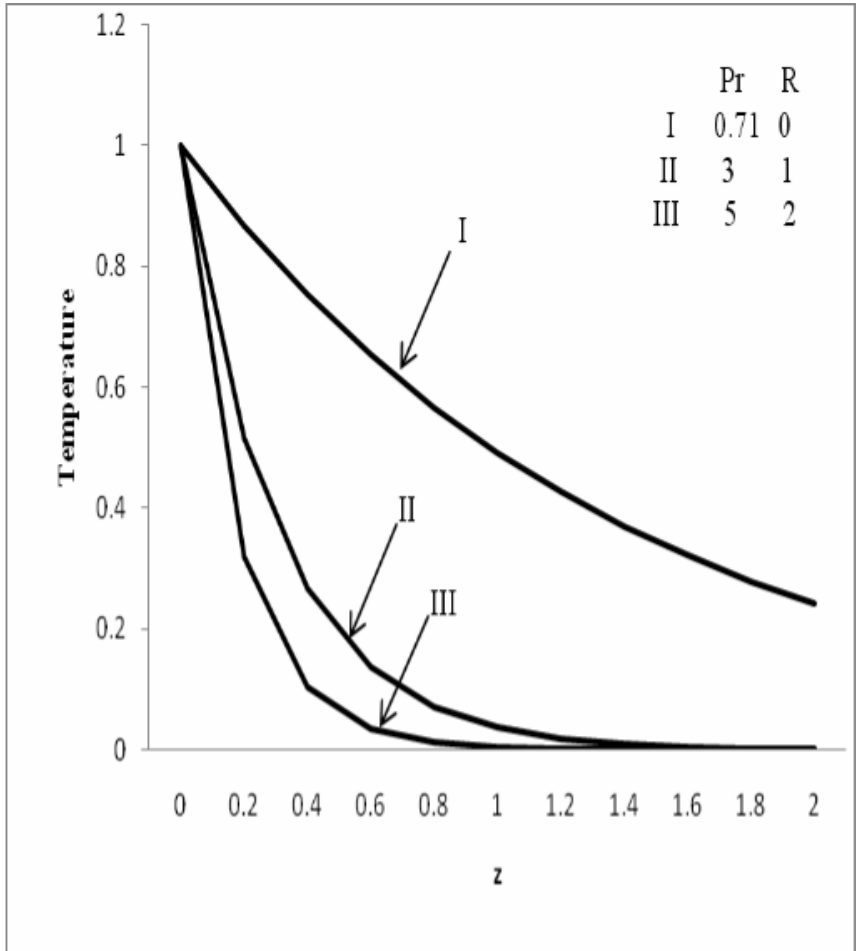

Fig. 3: Temperature profiles for $\varepsilon=0.001, \omega=2, t=1$, $A=0.1$ and simultaneous variations of values of Prandtl number, Pr and radiation parameter, $\mathbf{R}$.

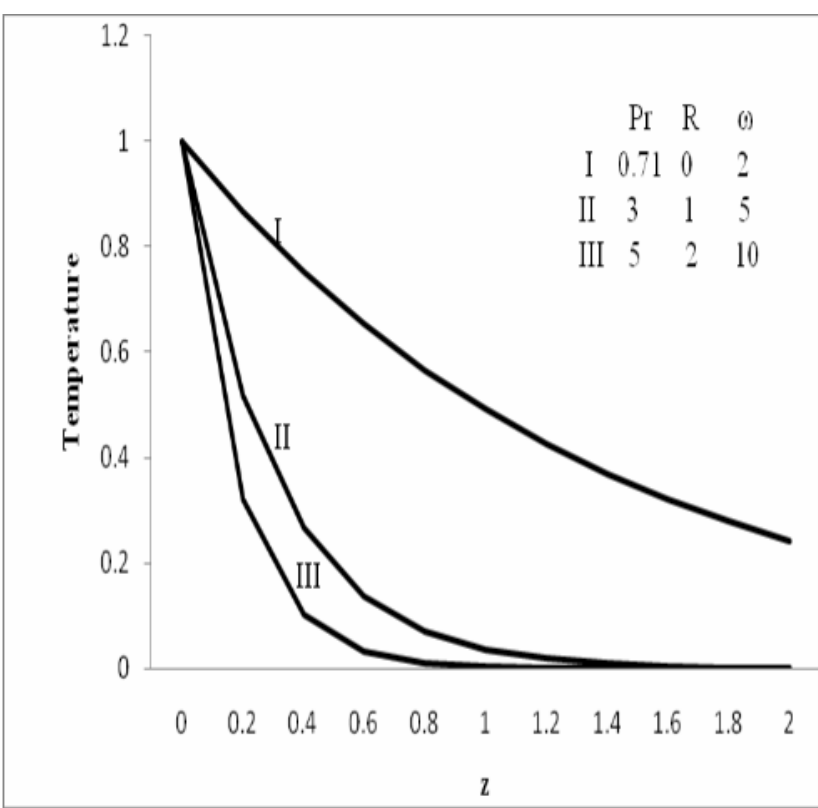

Fig. 4: Temperature profiles for $\varepsilon=0.01, t=1, A=0.1$ and simultaneous variations of values of Prandtl number, $\operatorname{Pr}$, frequency, $\omega$ and radiation parameter, $R$. 


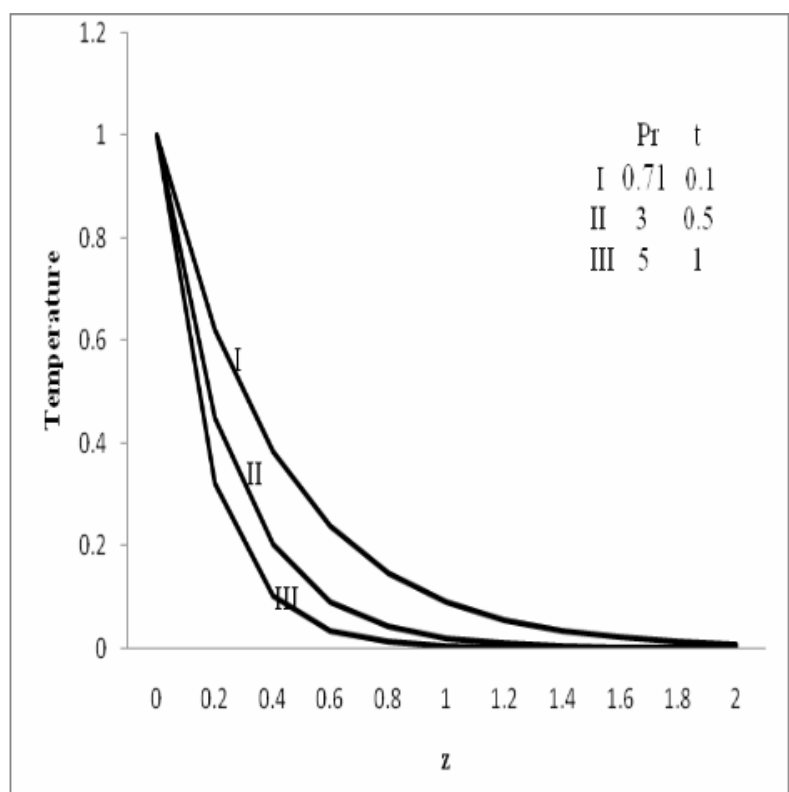

Fig. 5: Temperature profiles for $\varepsilon=0.01, R=2, \omega=2, A=$ 0.1 and simultaneous variations of values of time, $t$ and Prandtl number, Pr.

In figures 2-5 we presented the behaviour of the temperature for different values of flow parameters. Figure 2 shows that increase in the Prandtl number resulted to a decrease in temperature which converges close to the free steam value. Figure 3 revealed that in addition to increased Prandtl number, an increase in radiation parameter also led to decrease in temperature. This result corresponds to those of Isreal-Cookey et al [4], [5], [6], Soundalgekar [7], [8], [9] and Alagoa [10]. Figure 4 shows the temperature profiles for the simultaneous increase in Prandtl number, Pr, radiation parameter, $\mathrm{R}$, and frequency. It revealed that a simultaneous increase in these three parameters equally led to decrease in the temperature. Finally, figure 5 shows that a simultaneous increase in the Prandtl number and time resulted to decrease in temperature.

\section{CONCLUSION:}

In this study, an analytical close form approximate solution is obtained for the problem of temperature of vegetative ground in presence of time-dependent suction considering the effects of radiation parameter, prandtl number and frequency. The analysis and discussion of the results have been done. The study shows that the temperature of vegetative ground decreases with increase in the radiation parameter and prandtl number. Simultaneous increase in material parameters also decreased the temperature.
ACKNOWLEDGEMENT: All glory is to the most high God to whom knowledge and wisdom belong. My great appreciation is also due to $\mathrm{Dr} \mathrm{C}$. Isreal-Cookey for mentoring me into the studies of fluid flow and to my friend, Bola for errorless typesetting of the manuscript.

\section{REFERENCES}

(1)Tarkhar, H.S., Gorla, R.S.R. and Soundalgekar, V.M. "Radiation effects on MHD free Convection flow for a radiating gas past a semi-infinite vertical plate" , Int. J. Num. Meth. Heat Fluid flow 6,(2) 77-83, 1996.

(2)Liang, X, Wood, E.F., Land Lettemaler, D.P. Modelling ground heat flux in land surface parameterization schemes, Journal of Geophysical Research, 104, 95819600, 1999.

(3)Smith, E.A, Corson, W.L. and Tanner, B.D. Estimation of surface heat and moisture fluxes over prairie grassland in site energy budget measurements incorporating a cooled mirror dew point hygrometer, Journal of Goephysical Research. 97:18, 557-18,582, 1992.

(4)Isreal-Cookey, C. and Tay , G. "Transient flow of a radiating hygromagnetic fluid past an infinite vertical plate" $B$, AMSE, Vol. 7, No. 3, 4, 1-14, 2002.

(5)Isreal-Cookey, C. and Sigalo, F.B. "Unsteady freeconvection and mass transfer flow past an infinite heated porous vertical plate with time dependent suction" B, AMSE, Vol. 7,No. 3, 25-38, 2003.

(6)Isreal-C ookey, C. Ogulu, A., Omubo-Pepple, V.B. FreeConvection and mass transfer flow of a binary fluid considering the thermodiffusion and the diffusion-thermal effects and a tansverse magnetic field. Journal of Inst. Of Math \& Comp. Sci. (Math. Ser.) Vol. 21,No. 1, 1120,2008

(7)Soundalgekar, V.M. "Free Convectioneffects on the oscillatory flow past an infinite vertical porous plate with constant suction 1, Proc. Royal soc. London A 333, 25-36, 1973.

(8)Soundalgekar, V.M. "Free Convection effects on the oscillatory flow past an infinite vertical porous plate with constant suction 11, Proc. Royal soc. London A 333, 3750, 1973.

(9)Soundalgekar, V.M. "Free Convection effects on steady MHD flow past a vertical porous plate", J. Fluid Mechanics 66, 541-551,1974.

(10)Alagoa, K.D. "Transient flow of a magnetized plasma due to thermal perturbation", Astrophysics and space science. 254: 159-172,1997.

(11)Choudhury, B.J., Idso, S.B. and Reginato, R.J. "Analysis of an empirical model for soil heat flux under a growing wheat crop for estimating evaporation by an infraredtemperature based on energy balance equation" Agric for meteorol, 39, 283-297,1987.

(12)Wolfran, S. "Mathematica: A System of Doing Mathematics by Computers", Addison Wesley Publishing, England, 1991. 